\title{
Identificando Sinais de Estresse Pós-traumático Utilizando Dados Fisiológicos e Técnicas de Regressão
}

\author{
Luiz Antonio da Ponte Junior ${ }^{1,2}$, Débora Christina Muchaluat-Saade ${ }^{1,2}$, \\ Alexandre Plastino ${ }^{2}$, Rita de Cássia Alves ${ }^{3}$, Liana Catarina Lima Portugal ${ }^{3}$, \\ Leticia de Oliveira $^{3}$, Mirtes Garcia Pereira ${ }^{3}$
}

Laboratório MídiaCom ${ }^{1}$, Instituto de Computação ${ }^{2}$

Universidade Federal Fluminense, UFF

Niterói, Brasil

\{luizponte, debora\}@midiacom.uff.br, plastino@ic.uff.br

Laboratório de Neurofisiologia do Comportamento ${ }^{3}$, Instituto Biomédico

Universidade Federal Fluminense, UFF

Niterói, Brasil

\{cassiasalves, lianalportugal\}@gmail.com, \{oliveira_leticia, mirtes_pereira\}@id.uff.br

\begin{abstract}
Several studies propose the application of computing techniques to healthcare, in order to help doctors with diagnoses. This paper proposes the use of data mining to identify signs of post-traumatic stress based on physiological data. To evaluate the proposal, this work used a dataset of individuals who experienced traumatic events. These individuals had their heart rate and skin conductance collected during image visualization containing violent and non-violent scenes. Regression algorithms were applied to predict the individuals' PCL-C scale values and to identify a possible correlation between the signals and the scale. The IBk algorithm $(k=4)$, obtained the best correlation coefficient 0.4164 ( $p$-value $=0.001)$.
\end{abstract}

Resumo. Diversos estudos propõem aplicar técnicas computacionais à área da saúde para auxiliar médicos em seus diagnósticos. Este artigo propõe aplicar mineração de dados para identificar sinais de estresse pós-traumático baseado em dados fisiológicos. Para avaliar a proposta, foram usados dados de indivíduos que vivenciaram eventos traumáticos. Estes indivíduos tiveram seus sinais de frequência cardíaca e condutância da pele coletados durante a visualização de imagens com e sem cenas violentas. Foram utilizados algoritmos de regressão para predizer os valores da escala PCL-C dos indivíduos e identificar uma possível correlação entre os dados e a escala. O algoritmo IBk $(k=4)$ obteve o melhor coeficiente de correlação 0,4164 ( $p$-value $=0,001)$.

\section{Introdução}

A incidência de transtornos psicológicos tem aumentado em nossa sociedade. Durante a sua vida, um indivíduo pode vir a desenvolver um desses transtornos. A perda de um ente querido, ter sido vítima de um assalto ou tê-lo presenciado são exemplos de situações e fatores que colaboram para o surgimento de um transtorno psicológico.

Um dos transtornos psicológicos, e foco de estudo deste trabalho, é o Transtorno do Estresse Pós-Traumático (TEPT). Esse transtorno acomete indivíduos que sofreram 
algum evento traumático em suas vidas. Assim como outros transtornos, o TEPT provoca reações neurofisiológicas no corpo do indivíduo, como aumento da pressão sanguínea, alteração da condutância da pele, taquicardia e bradicardia. Ao vivenciar eventos que remetam aos traumas passados, o indivíduo é acometido por tais reações neurofisiológicas.

O correto diagnóstico do TEPT, e de qualquer transtorno, é fundamental para a eficiência e eficácia do tratamento. Uma das ferramentas utilizadas por médicos e especialistas no auxílio ao diagnóstico do TEPT é o uso de questionários que avaliam o grau de sintomas de TEPT de acordo com uma escala pré-definida. Uma dessas escalas é a Posttraumatic Stress Disorder Checklist (PCL), que em sua quarta versão possui três variantes: PCL-M (military), desenvolvida para militares, PCL-C (civilian), desenvolvida para civis e PCL-S (specific), designada a um evento traumático específico [Weathers et al. 1993].

A escala PCL seleciona possíveis portadores de TEPT, fornecendo um diagnóstico provisório, capaz de auxiliar médicos e especialistas. Ela pode ser empregada para acompanhar o tratamento como forma de avaliar a mudança e o desenvolvimento dos sintomas.

Alguns trabalhos [Karstoft et al. 2015, Saxe et al. 2017] aplicam Inteligência Artificial a fim de identificar a existência de uma correlação entre dados de saúde de indivíduos diagnosticados com TEPT, ou que foram submetidos a algum evento traumático, e os valores de escalas que avaliam os sintomas do TEPT. Sendo assim, tais trabalhos não só auxiliam o diagnóstico e a prevenção de transtornos, como também propõem novos mecanismos mais simples e econômicos, capazes de identificar seus sintomas.

O objetivo geral deste trabalho é identificar sinais de TEPT utilizando dados fisiológicos e técnicas de mineração de dados. Um experimento realizado no Instituto Biomédico da Universidade Federal Fluminense (UFF), aprovado pelo Comitê de Ética (parecer de número 203/09), coletou os sinais fisiológicos de frequência cardíaca e condutância da pele de voluntários que vivenciaram eventos traumáticos. Os sinais foram coletados durante a visualização de imagens de estímulos emocionais e neutros e, ao final, o questionário da escala PCL-C foi aplicado.

Os objetivos específicos deste trabalho são: criar e avaliar modelos de regressão capazes de predizer o valor da escala de PCL-C a partir dos sinais fisiológicos dos indivíduos e estudar a existência de uma correlação entre estes sinais e os valores da escala de PCL-C. Pelo fato desses sinais serem fáceis de coletar, isto é, os sensores utilizados são de baixo custo, possuem um tamanho pequeno e não são invasivos, o seu uso pode ser uma alternativa barata e viável aos exames existentes para o diagnóstico de TEPT.

Este trabalho está estruturado da seguinte forma. A Seção 2 apresenta os trabalhos relacionados. A Seção 3 aborda os conceitos principais utilizados neste trabalho. A Seção 4 explica como os dados utilizados foram coletados. A Seção 5 apresenta os resultados obtidos com os algoritmos de regressão e a Seção 6 resume as conclusões obtidas.

\section{Trabalhos Relacionados}

Diversos trabalhos aplicam técnicas computacionais à área da saúde, realizando análises estatísticas em dados de indivíduos portadores de algum transtorno ou doença, a fim de auxiliar médicos e especialistas com o diagnóstico e prevenção de doenças e transtornos. Esses dados se referem a características do indivíduo (e.g., idade, sexo, peso), a hábitos comportamentais (e.g., fumar, beber, praticar atividade física) ou a respostas e pontuações 
de questionários e escalas de medição de sintomas, por exemplo. Os resultados desses trabalhos comprovam a importância da computação e apresentam as suas contribuições para a área da saúde.

O trabalho [Omurca and Ekinci 2015] propõe um sistema inteligente híbrido que combina algoritmos de classificação e seleção de atributos para classificar indivíduos com risco de desenvolver TEPT. Nas análises, foi utilizada uma base de dados com 391 indivíduos, dos quais 321 possuem risco de desenvolver TEPT e 70 não possuem risco. Foram utilizados os classificadores SMO, Multilayer Perceptron e Nä̈ve Bayes, que apresentaram acurácias entre 74 e $79 \%$.

Os trabalhos [Karstoft et al. 2015, Galatzer-Levy et al. 2014] utilizaram uma base de dados com 957 sobreviventes a eventos traumáticos ocorridos próximos à data de admissão ao departamento de emergência do hospital. Os sobreviventes foram monitorados por 15 meses, tendo seus dados coletados na admissão ao hospital e nos $7^{\circ}$ e $15^{\circ}$ meses após a admissão. Os autores aplicaram os algoritmos SVM (Support Vector Machine), Random Forest e Kernel Ridge Regression, juntamente com seleção de atributos, a fim de encontrar o subconjunto mínimo de atributos que maximizasse a predição de TEPT. Os resultados encontrados suportam a hipótese da existência de múltiplos conjuntos de fatores de risco associados ao TEPT.

Exames de imagens, como ressonância magnética (MRI) e eletroencefalograma (EEG), também são muito utilizados em pesquisas que procuram predizer e identificar biomarcadores ou fatores de risco em indivíduos portadores de algum transtorno psicológico. O trabalho [Lanius et al. 2002] analisa, por meio de ressonância magnética funcional (fMRI), as áreas de ativação cerebral em pacientes com TEPT que apresentam respostas dissociativas ao relembrar os eventos traumáticos vivenciados.

A resposta dissociativa consiste na perda de memória, consciência ou percepção do ambiente ao tentar se lembrar do acontecimento traumático, sendo considerada um mecanismo involuntário de defesa do organismo a fim de preservar o indivíduo. Indivíduos que apresentam respostas dissociativas não lembram com exatidão os detalhes do evento. Os resultados de [Lanius et al. 2002] revelam que os indivíduos com TEPT apresentaram maior ativação cerebral em determinadas regiões em comparação aos indivíduos do grupo de controle (i.e., indivíduos sadios).

No trabalho [Portugal et al. 2016], os autores aplicaram técnicas de regressão, como o algoritmo Relevance Vector Regression, e reconhecimento de padrão em imagens fMRI de 57 jovens para determinar a desregulação comportamental e emocional dos participantes. $\mathrm{O}$ algoritmo conseguiu identificar padrões de atividade neural associados à desregulação, indicando as regiões cerebrais com maior contribuição.

Frequência cardíaca, condutância da pele e pressão sanguínea são alguns dos tipos de dados fisiológicos que costumam ser coletados durante a realização de experimentos com indivíduos portadores de transtornos psicológicos. Esses dados, em comparação aos dados de imagem, geralmente são mais acessíveis e fáceis de serem coletados devido à simplicidade dos equipamentos e sensores disponíveis no mercado.

O trabalho [Shalev et al. 1998] estuda o relacionamento entre a frequência cardíaca e a pressão sanguínea coletadas imediatamente após um evento traumático e o subsequente desenvolvimento de TEPT. Nesse trabalho, 86 sobreviventes a eventos 
traumáticos foram monitorados por quatro meses, tendo os seus dados e sintomas coletados e medidos ao chegar ao hospital, na $1^{\mathrm{a}}$ semana, no $1^{\circ}$ mês e no $4^{\mathrm{o}}$ mês após a chegada. Dos 86 indivíduos, 20 foram diagnosticados com TEPT no $4^{\circ}$ mês. Os resultados do trabalho mostram que os 20 indivíduos diagnosticados com TEPT apresentaram altas frequências cardíacas na chegada ao hospital e na $1^{\mathrm{a}}$ semana, em comparação aos demais indivíduos. Em contrapartida, a pressão sanguínea não diferiu.

Uma pesquisa identificou que mulheres portadoras de TEPT tem 1,4 vezes mais chances de desenvolver dependência alcoólica [Regier et al. 1990]. Em [Harrington and Newman 2007], os autores analisam as escalas Penn Inventory e PCL-C a fim de encontrar um ponto de corte que maximize a predição de mulheres dependentes de substâncias e que atendam aos critérios de diagnóstico de TEPT.

Em [Leightley et al. 2019], os autores aplicaram algoritmos de classificação a uma base de dados de 13690 militares do Reino Unido. Além de conter apenas dados demográficos, a base possuía a pontuação do questionário da escala PCL. Apesar de os indivíduos serem militares, foi aplicada a versão civil da escala PCL, utilizando o valor 50 como ponto de corte. Segundo os autores, a escala PCL-C foi selecionada por ser menos restritiva em populações que podem ter vivenciado eventos traumáticos não relacionados às missões militares. Os autores aplicaram SVM, Random Forest, Redes Neurais Artificiais e Bagging, apresentando, respectivamente, acurácias iguais a 0,91, 0,97, 0,89 e 0,95. Uma das limitações apontadas é o desbalanceamento da base, pois apenas 3,95\% dos indivíduos correspondem a possíveis portadores de TEPT.

A partir de uma pesquisa a trabalhos relacionados à predição de indivíduos portadores de TEPT ou vítimas de eventos traumáticos através da aplicação de técnicas de mineração de dados com o uso de dados fisiológicos e de escalas, pôde-se verificar que a escala PCL ainda não foi amplamente explorada.

A maioria dos trabalhos encontrados faz uso de outras escalas (e.g., BDI, CAPS, IES, STAI e SCID) referentes aos sintomas de TEPT. Sendo assim, este trabalho explora o uso da escala PCL-C juntamente com dados fisiológicos de frequência cardíaca e condutância da pele. Através da aplicação de algoritmos de regressão para predizer os valores da escala PCL-C, avalia-se a existência de uma correlação entre a escala e esses dados.

\section{Fundamentação Teórica}

Nesta seção, serão apresentados os conceitos utilizados neste trabalho. As Subseções 3.1 e 3.2 contextualizam respectivamente o Transtorno do Estresse Pós-Traumático e a escala PCL utilizada para medir o grau de sintomas desse transtorno. A Subseção 3.3 explica o conceito de regressão em mineração de dados. As Subseções 3.4 e 3.5 explicam respectivamente os métodos de validação e de avaliação de significância estatística.

\subsection{Transtorno do Estresse Pós-Traumático (TEPT)}

O transtorno do estresse pós-traumático está incluso no Manual Diagnóstico e Estatístico de Transtornos Mentais (Diagnostic and Statistical Manual of Mental Disorders (DSM)) [Association et al. 2013], elaborado pela Associação Americana de Psiquiatria. Segundo o DSM, o TEPT é um transtorno psiquiátrico que pode se desenvolver em pessoas que vivenciaram ou testemunharam um evento traumático. 
É importante notar que nem todas as pessoas que vivenciam eventos traumáticos desenvolvem TEPT. O impacto e a recorrência do evento vivenciado estão ligados ao desenvolvimento do transtorno. Para receber o diagnóstico de TEPT, os sintomas devem durar por mais de um mês e persistir por um longo tempo (meses e até mesmo anos). Além disso, o TEPT costuma ocorrer juntamente com outras condições, como depressão, transtornos de ansiedade e estresse, e abuso de substâncias químicas e alcoólicas.

Portadores de TEPT costumam ter pensamentos intrusivos e sentimentos relacionados ao evento vivenciado. Ao reviver o evento através de lembranças ou até mesmo de situações semelhantes, diversas reações neurofisiológicas são provocadas, causando variações na frequência cardíaca, condutância da pele e pressão sanguínea, por exemplo.

\subsection{Posttraumatic Stress Disorder Checklist (PCL)}

A escala PCL é um instrumento desenvolvido pelo Veterans Affairs National Center for PTSD que faz uma triagem dos possíveis portadores de TEPT, fornecendo um diagnóstico provisório. Essa escala também pode ser usada para monitorar a mudança e o desenvolvimento dos sintomas durante e após o tratamento [Weathers et al. 1993].

A escala PCL se fundamenta nos sintomas de TEPT descritos pelo DSM [Association et al. 2013]. À medida que o DSM é revisado, a escala PCL também costuma ser atualizada. Atualmente, a versão mais recente da escala PCL é a PCL-5 [Weathers et al. 2013], resultado da quinta revisão do DSM. Neste trabalho, entretanto, foi aplicada a versão PCL-C (civil) da escala PCL-4, referente ao DSM-IV.

A versão PCL-C da escala PCL-4 é composta de um questionário de 17 perguntas referentes a problemas comumente apresentados em resposta ao trauma vivenciado. Para cada pergunta, o participante avalia determinado problema ocorrido no mês anterior à realização do questionário, definindo uma pontuação de 1 a 5, onde 1 corresponde ao menor grau de incômodo causado pelo problema e 5 corresponde ao maior grau.

Os valores da escala vão de 17 a 85 de acordo com o Veterans Affairs $\mathrm{Na}$ tional Center for PTSD. Existem estudos que sugerem determinados pontos de corte para atender a critérios de classificação ou identificação de portadores de TEPT em diagnósticos provisórios ${ }^{1}$. Neste trabalho, apesar de não terem sido aplicados algoritmos de classificação, foi utilizado o valor 44 como ponto de corte para avaliar quantas instâncias possuem valores iguais ou superiores a 44 , indicando possíveis portadores de TEPT. Com isso, pode-se observar que há um desbalanceamento da base de dados, pois apenas 8 das 55 instâncias possuem $P C L \geq 44$.

\subsection{Regressão}

A regressão é um tipo de metodologia estatística utilizada em mineração de dados para realizar predições de valores numéricos. As análises de regressão são compostas de duas etapas: aprendizagem e predição. Durante a etapa de aprendizagem, um modelo é construído através de uma função matemática gerada com base nos valores conhecidos de um subconjunto da base de dados original, chamado de partição de treinamento.

Cada instância da partição de treinamento possui, além de seus atributos independentes, o atributo dependente a ser predito. $\mathrm{O}$ modelo é o responsável pela atribuição do

\footnotetext{
${ }^{1}$ https://www.ptsd.va.gov/professional/assessment/documents/PCL_ handoutDSM4 $\cdot$ pdf
} 
valor do atributo dependente de uma instância, a partir dos valores dos atributos independentes. Esta etapa de aprendizagem também pode ser vista como uma descoberta de um conjunto de regras ou de uma função $y=f(x)$ que mapeia o valor $x$ de uma instância ao seu valor $y$, resultando em sua predição.

Durante a etapa de predição, o modelo construído e treinado na etapa de aprendizagem é utilizado para realizar as predições do valor do atributo dependente de novas instâncias. Nesta etapa, o modelo realizará as predições aplicando a função matemática obtida em um conjunto ou partição de teste, composto pelas instâncias da base de dados original que não estavam na partição de treinamento. Com isso, é possível avaliar corretamente o desempenho de predição do modelo, já que este não utilizou previamente essas instâncias em sua etapa de aprendizagem.

Dentre os algoritmos de regressão amplamente utilizados, foram empregados nas análises de regressão deste artigo: k-NN (k-Nearest-Neighbor), SVM (Support Vector Machine) e Linear Regression.

O algoritmo k-NN considera cada instância da partição de treino, descrita por $n$ atributos, como um ponto em um espaço de $n$ dimensões. Ao receber uma instância da partição de teste, o k-NN procura as $k$ instâncias de treino do espaço $n$-dimensional que são mais próximas da instância de teste. A predição é realizada a partir da média dos valores dos atributos dependentes das $k$ instâncias de treino. Dentre os parâmetros que este algoritmo possui, destaca-se o parâmetro $k$, que representa a quantidade de instâncias de treino do espaço $n$-dimensional a serem buscadas. Nas análises de regressão, este parâmetro foi variado com valores de 1 a 15 .

O algoritmo SVM utiliza um mapeamento não linear para transformar os dados originais da partição de treino, que já estão num espaço dimensional, em um outro espaço dimensional maior, de modo que os dados possam ser separados por um hiperplano. $\mathrm{O}$ SVM procura então pelo hiperplano linear ótimo, que melhor separa as instâncias. Um dos parâmetros deste algoritmo é o kernel, responsável por definir a função de kernel aplicada para transformar os dados em um espaço dimensional maior. Nas análises de regressão, esse parâmetro utilizou a função de kernel PolyKernel.

O algoritmo Linear Regression modela o atributo dependente (y) como uma função linear dos atributos independentes $(x)$ da base de dados, conforme indica a Equação 1.

$$
y=w x+b
$$

Na equação, $y$ e $x$ são, respectivamente, os atributos dependente e independentes, $w$ é o coeficiente de regressão e $b$ é o componente estocástico, que representa possíveis erros e desvios.

\subsection{Método de Validação}

Conforme explicado na Subseção 3.3, a análise de regressão é composta por duas etapas: a etapa de aprendizagem e a etapa de predição. Durante a etapa de aprendizagem, são geradas as partições de treinamento e de teste. A partição de treinamento será utilizada na primeira etapa (etapa de aprendizagem), enquanto a partição de teste será utilizada na 
segunda etapa (etapa de predição). Esse particionamento da base de dados é fundamental para se avaliar a capacidade de predição do modelo gerado. O modo como as partições são geradas pode diferir de acordo com o método de validação empregado.

Neste trabalho, foi empregado o método k-Fold Cross-Validation. Nele, a base de dados inicial é randomicamente particionada em $k$ partições ou folds mutualmente exclusivos $F_{1}, F_{2}, F_{3}, \ldots, F_{k}$, onde cada fold possui aproximadamente o mesmo tamanho. São executadas $k$ iterações. Na iteração $i$, o fold $F_{i}$ é destinado para o teste e os demais folds são utilizados para o treinamento do modelo. Neste método, cada instância é utilizada o mesmo número de vezes $(k-1)$ para treinamento e uma única vez para teste.

\subsection{Teste de Significância Estatística}

Após a construção de um modelo e da obtenção de suas estimativas de coeficiente de correlação e demais métricas, deseja-se saber se tais resultados são estatisticamente significantes, i.e., se eles não ocorreram ao acaso. Um dos testes de significância estatística é o teste de permutação. Nele, são gerados $n$ bases de dados, onde os valores do atributo a ser predito de cada instância são permutados entre si.

Para cada uma das $n$ bases de dados permutadas, são executadas as etapas de aprendizagem e de predição, obtendo-se as estimativas das métricas. Em seguida, comparam-se as métricas obtidas através da base de dados inicial com as métricas obtidas em cada base de dados permutada, calculando a porcentagem de resultados melhores ou iguais aos resultados da base de dados inicial. Essa porcentagem calculada para uma métrica representa o $p$-value do teste de permutação. Por fim, estabelece-se um nível de significância $\alpha$ como limiar para avaliar os resultados. Na prática, utiliza-se $\alpha=0,05$ ou $\alpha=0,01$. Os resultados obtidos são considerados significativos, se $\mathrm{p}$-value $\leq \alpha$.

A ferramenta WEKA (versão 3.8.3) [Witten et al. 2016] utilizada nas análises de regressão não implementa o teste de permutação para avaliar a significância estatística dos resultados de seus algoritmos de regressão e de classificação. Portanto, além das análises de regressão realizadas neste trabalho, foi implementado o algoritmo do teste de permutação para a API da ferramenta WEKA, visando estender sua funcionalidade.

\section{Aquisição dos Dados}

A base de dados utilizada neste trabalho foi obtida por meio de um experimento realizado no Instituto Biomédico da Universidade Federal Fluminense (UFF) [Alves 2012], aprovado pelo Comitê de Ética em Pesquisa do Hospital Universitário Antônio Pedro (HUAP) (parecer de número 203/09). O experimento coletou os sinais fisiológicos de frequência cardíaca (FC) e condutância da pele de voluntários que sofreram algum evento traumático relacionado à violência. O sinal de condutância da pele mede a resposta da variação elétrica da pele. Essa variação elétrica é medida por meio do chamado Número de Respostas (NR), cuja unidade de medida é micro Siemens $(\mu \mathrm{S})$. Após a coleta dos dados fisiológicos, os participantes responderam o questionário da versão 4 da escala PCL-C para avaliar o seu grau de sintomas de TEPT.

O experimento foi realizado com 83 estudantes voluntários, que foram vítimas de algum acontecimento traumático. Dentre os 83 voluntários, aqueles que faziam uso de algum medicamento com ação no sistema nervoso central ou que apresentaram arritmias 
cardíacas foram removidos da base de dados. Como resultado, a base de dados utilizada passou a conter 55 voluntários.

O experimento teve como objetivo investigar a resposta autonômica da frequência cardíaca e da condutância da pele provocada pela visualização de imagens relacionadas à violência e explorar como a direcionalidade do estímulo e a vivência de eventos traumáticos influenciam a reatividade cardíaca do indivíduo. As imagens foram divididas em blocos de cenas de violência direcionada (VD) e violência não direcionada (VND). Cada bloco continha 16 imagens de estímulo emocional e 16 de estímulo neutro, totalizando 32 imagens por bloco.

Imagens de violência direcionada (VD) consistem em uma pessoa segurando uma arma direcionada ao espectador, pertencendo às imagens de estímulo emocional do bloco direcionado. Imagens neutras de violência direcionada (NeuVD) consistem em uma pessoa segurando um objeto (e.g., câmera, guarda-chuva e microfone) direcionado ao espectador, pertencendo às imagens de estímulo neutro do bloco direcionado.

Imagens de violência não direcionada (VND) consistem em uma pessoa segurando uma arma direcionada a terceiros, pertencendo às imagens de estímulo emocional do bloco não direcionado. Imagens neutras de violência não direcionada (NeuVND) contêm alguém segurando um objeto (e.g., câmera, guarda-chuva e microfone) direcionado a terceiros, pertencendo às imagens de estímulo neutro do bloco não direcionado.

As imagens foram exibidas por 6 segundos, sendo intercaladas por uma cruz de fixação localizada no centro da tela e exibida por 6 a 8 segundos. As imagens foram exibidas de forma pseudo-aleatória dentro de seus blocos a fim de evitar que muitas imagens de mesmo estímulo fossem apresentadas em sequência. A Figura 1 ilustra como a visualização dos blocos de imagens é realizada no experimento.

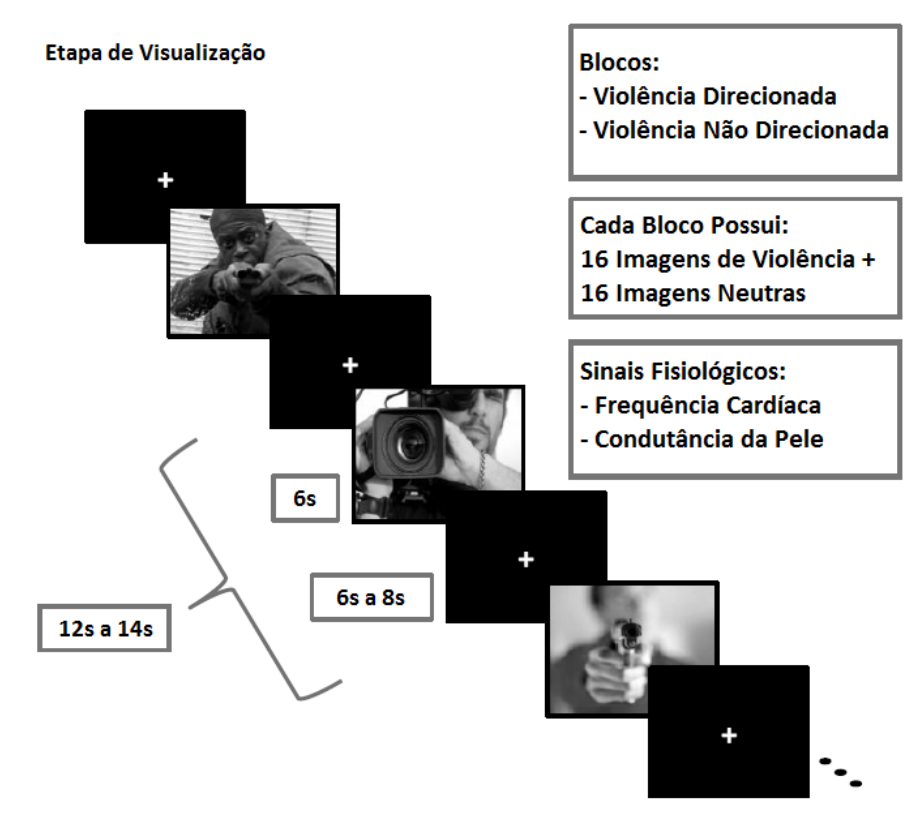

Figura 1. Visualização dos Blocos de Imagens do Experimento [Alves 2012]

A visualização de cada bloco de imagens durava aproximadamente 7 minutos. Em seguida, os voluntários eram solicitados a preencher o questionário da escala PCL-C. 
A base de dados deste trabalho contém cinco atributos referentes aos sinais fisiológicos de frequência cardíaca (FC), cinco atributos referentes aos sinais de condutância da pele e um atributo referente ao valor da escala PCL-C, totalizando 11 atributos. A Tabela 1 exibe os atributos pertencentes à base de dados.

\begin{tabular}{|c|c|c|c|}
\hline Atributo & Tipo de Sinal & Valor Mínimo & Valor Máximo \\
\hline VD & Frequência Cardíaca & $-4,81$ & 2,465 \\
\hline VND & Frequência Cardíaca & $-5,179$ & 3,674 \\
\hline NeuVD & Frequência Cardíaca & $-2,91$ & 3,436 \\
\hline NeuVND & Frequência Cardíaca & $-3,167$ & 2,048 \\
\hline NR_VD & Condutância da Pele & 0 & 8 \\
\hline NR_VND & Condutância da Pele & 0 & 11 \\
\hline NR_NeuVD & Condutância da Pele & 0 & 5 \\
\hline NR_NeuVND & Condutância da Pele & 0 & 8 \\
\hline FC_Viol-Neutra & Frequência Cardíaca & $-3,517$ & 2,622 \\
\hline NR_Viol-Neutra & Condutância da Pele & -3 & 7 \\
\hline
\end{tabular}

Tabela 1. Descrição dos Atributos Independentes

$\mathrm{O}$ atributo VD contém a média de valores do sinal de frequência cardíaca (FC) coletados durante os 6 segundos (12 pontos de amostra, cada um com 0,5 segundo) de visualização de todas as 16 imagens de violência direcionada (VD). Em outras palavras, primeiro é calculada a média dos 12 pontos de amostra de uma imagem de VD, resultando na FC média daquela imagem. Em seguida, este cálculo é aplicado para o restante das imagens de VD. Neste momento, existem 16 valores médios de FC (um para cada imagem de VD). Por último, é calculada a média desses 16 valores de FC. Os atributos VND, NeuVD e NeuVND contêm o mesmo cálculo descrito para o atributo VD, utilizando os valores do sinal de FC coletados durante a visualização das imagens correspondentes.

Analogamente, o atributo NR_VD contém a média dos valores de NR (i.e., resposta da variação elétrica da pele) coletados durante os 6 segundos (12 pontos de amostra, cada um com 0,5 segundo) de visualização de todas as 16 imagens de VD. Os atributos NR_VND, NR_NeuVD e NR_NeuVND contêm o mesmo cálculo descrito para o atributo NR_VD, utilizando os valores de NR coletados durante a visualização das imagens correspondentes.

O atributo FC_Viol-Neutra contém a subtração do estímulo neutro (NeuVD e NeuVND) do estímulo emocional (VD e VND) dos valores do sinal de FC. O cálculo é realizado por meio da Equação 2.

$$
F C_{-} \text {Viol-Neutra }=\frac{(\mathrm{VD}+\mathrm{VND})}{2}-\frac{(\mathrm{NeuVD}+\mathrm{NeuVND})}{2}
$$

O atributo NR_Viol-Neutra contém a subtração do estímulo neutro (NR_NeuVD e NR_NeuVND) do estímulo emocional (NR_VD e NR_VND) dos valores de NR. O cálculo é realizado por meio da Equação 3.

$$
N R \_ \text {Viol-Neutra }=(\text { NR_VD }+ \text { NR_VND })-(\text { NR_NeuVD }+ \text { NR_NeuVND })
$$




\section{Análises de Regressão}

Esta seção discute as análises de regressão realizadas com a ferramenta WEKA (versão 3.8.3) [Witten et al. 2016], apresentando os resultados obtidos. Conforme explicado na Subseção 3.3, foram utilizados os seguintes algoritmos da WEKA: IBk (implementação do algoritmo k-NN na WEKA), SMOreg (implementação do algoritmo SVM para regressão na WEKA) e Linear Regression.

As análises foram realizadas utilizando o método 5-Fold Stratified CrossValidation, obtendo os resultados para as métricas coeficiente de correlação (R), erro absoluto médio (mean absolute error ou MAE) e raiz do erro quadrático médio (root mean squared error ou RMSE). Para avaliar os resultados obtidos, foi aplicado o teste de permutação, implementado para a WEKA, realizando 1000 permutações e utilizando $\alpha=0,05$ como nível de significância.

O algoritmo IBk possui o parâmetro $k$, que representa a quantidade de vizinhos mais próximos da instância de teste a serem identificados. Nas análises realizadas, o parâmetro $k$ variou de 1 a 15 . A Tabela 2 contém os resultados obtidos com os três algoritmos utilizados.

\begin{tabular}{|c|c|c|c|c|c|c|}
\hline Algoritmo & $\mathrm{R}$ & $\mathrm{R}$ p-value & MAE & MAE p-value & RMSE & RMSE p-value \\
\hline IBk k $=1$ & 0,3808 & 0,005 & 10,2364 & 0,021 & 14,2184 & 0,023 \\
\hline IBk k $=2$ & 0,4031 & 0,001 & 9,6636 & 0,029 & 12,583 & 0,014 \\
\hline IBk k $=3$ & 0,4055 & 0,001 & 9,1394 & 0,012 & 11,9894 & 0,004 \\
\hline IBk k $=4$ & 0,4164 & 0,001 & 8,9636 & 0,013 & $\mathbf{1 1 , 5 1 8 6}$ & 0,001 \\
\hline IBk k $=5$ & 0,3874 & 0,001 & 8,8691 & 0,007 & 11,6159 & 0,001 \\
\hline Ibk k $=6$ & 0,3232 & 0,005 & 9,3455 & 0,05 & 11,9413 & 0,006 \\
\hline IBk k = 7 & 0,2616 & 0,025 & 9,561 & 0,103 & 12,1385 & 0,021 \\
\hline IBk k $=8$ & 0,1513 & 0,095 & 9,7795 & 0,189 & 12,5402 & 0,077 \\
\hline IBk k $=9$ & 0,014 & 0,307 & 10,1636 & 0,445 & 12,9528 & 0,253 \\
\hline IBk k $=10$ & $-0,0249$ & 0,384 & 10,1782 & 0,493 & 12,9678 & 0,303 \\
\hline IBk k $=11$ & $-0,1288$ & 0,613 & 10,4149 & 0,728 & 13,1805 & 0,514 \\
\hline IBk k $=12$ & $-0,1187$ & 0,585 & 10,3697 & 0,712 & 13,1396 & 0,524 \\
\hline IBk k $=13$ & $-0,0739$ & 0,459 & 10,1469 & 0,58 & 12,9559 & 0,381 \\
\hline $\mathrm{IBk} \mathrm{k}=14$ & $-0,0309$ & 0,355 & 9,9351 & 0,393 & 12,8605 & 0,322 \\
\hline IBk k $=15$ & 0,0096 & 0,258 & 9,8848 & 0,373 & 12,7532 & 0,242 \\
\hline Linear Regression & 0,0396 & 0,242 & 10,2669 & 0,375 & 13,4548 & 0,436 \\
\hline SMOreg & 0,3318 & 0,015 & 8,442 & 0,005 & 12,0681 & 0,013 \\
\hline
\end{tabular}

Tabela 2. Resultados das Análises de Regressão com a ferramenta WEKA

Conforme pode-se observar, o melhor resultado (em negrito) foi obtido com o algoritmo IBk e $k=4$, apresentando o maior coeficiente de correlação $(0,4164)$ e com significância estatística ( $p$-value $=0,001$ ). À primeira vista, o coeficiente de correlação obtido parece baixo. Entretanto, profissionais de saúde parceiros que investigam técnicas para propostas de novos marcadores para doenças psicológicas o consideraram promissor. Além disso, este resultado apresentou o terceiro menor MAE e o menor RMSE, ambos com significância estatística ( $p$-value $\leq 0,05)$. 


\section{Conclusões}

A aplicação da computação nas áreas da saúde para identificar portadores de transtornos e até mesmo candidatos a desenvolver tais transtornos é foco de diversos trabalhos e pesquisas. Além de promissora, tal aplicação tem auxiliado médicos e especialistas em diagnósticos mais eficientes e eficazes.

Conforme evidenciado anteriormente, este trabalho explora, de forma inédita, a escala PCL, aplicando técnicas de mineração de dados sobre dados fisiológicos de frequência cardíaca e condutância de pele. Enquanto os trabalhos [Bliese et al. 2008, Harrington and Newman 2007, Monson et al. 2008] avaliam a escala PCL em conjunto com dados demográficos, comparando seus resultados com os de outras escalas de TEPT, este trabalho explorou o uso da escala PCL com sinais fisiológicos para avaliar a existência de uma possível correlação entre eles.

O melhor resultado das análises de regressão obtidos com a ferramenta WEKA apresentou coeficiente de correlação (R) 0,4164, utilizando o algoritmo IBk com o seu parâmetro $k=4$. Apesar de o coeficiente de correlação parecer baixo, os profissionais de saúde parceiros que investigam técnicas para propostas de novos marcadores para doenças psicológicas consideraram promissor o resultado obtido na análise proposta neste artigo.

Como contribuição tecnológica adicional deste trabalho, destaca-se a implementação do teste de permutação para a ferramenta WEKA, visando adicionar uma forma de validação da significância estatística dos resultados obtidos com os algoritmos da ferramenta.

Como limitações enfrentadas estão as pequenas dimensões da base de dados, tanto na quantidade de instâncias, como no número de atributos, juntamente com o seu desbalanceamento, i.e., há poucas instâncias com valores de PCL considerados alto (valores $\geq 44$ ), afetando os resultados das análises de regressão. Como trabalhos futuros, pretende-se avaliar técnicas de classificação para prever o valor nominal (baixo ou alto) do atributo PCL, em vez de regressão, e também repetir o experimento com um número maior de indivíduos para reavaliar as técnicas adotadas com uma base maior.

\section{Referências}

Alves, R. d. C. S. (2012). Modulação da Frequência Cardíaca pela Visualização de Estímulos de Ameaça. Master's thesis, Biomedical Institute, Federal Fluminense University, Niterói, RJ, Brazil.

Association, A. P. et al. (2013). Diagnostic and statistical manual of mental disorders (DSM-5). American Psychiatric Pub, 5 edition.

Bliese, P. D., Wright, K. M., Adler, A. B., Cabrera, O., Castro, C. A., and Hoge, C. W. (2008). Validating the primary care posttraumatic stress disorder screen and the posttraumatic stress disorder checklist with soldiers returning from combat. Journal of Consulting and Clinical Psychology, 76(2):272.

Galatzer-Levy, I. R., Karstoft, K.-I., Statnikov, A., and Shalev, A. Y. (2014). Quantitative forecasting of PTSD from early trauma responses: A machine learning application. Journal of Psychiatric Research, 59:68-76. 
Harrington, T. and Newman, E. (2007). The psychometric utility of two self-report measures of PTSD among women substance users. Addictive Behaviors, 32(12):2788-2798.

Karstoft, K.-I., Galatzer-Levy, I. R., Statnikov, A., Li, Z., and Shalev, A. Y. (2015). Bridging a translational gap: using machine learning to improve the prediction of PTSD. BMC Psychiatry, 15(1):30.

Lanius, R. A., Williamson, P. C., Boksman, K., Densmore, M., Gupta, M., et al. (2002). Brain activation during script-driven imagery induced dissociative responses in PTSD: a functional magnetic resonance imaging investigation. Biological Psychiatry, 52(4):305-311.

Leightley, D., Williamson, V., Darby, J., and Fear, N. T. (2019). Identifying probable post-traumatic stress disorder: applying supervised machine learning to data from a UK military cohort. Journal of Mental Health, 28(1):34-41.

Monson, C. M., Gradus, J. L., Young-Xu, Y., Schnurr, P. P., Price, J. L., et al. (2008). Change in posttraumatic stress disorder symptoms: do clinicians and patients agree? Psychological Assessment, 20(2):131.

Omurca, S. İ. and Ekinci, E. (2015). An alternative evaluation of post traumatic stress disorder with machine learning methods. In 2015 International Symposium on Innovations in Intelligent SysTems and Applications (INISTA), pages 1-7. IEEE.

Portugal, L. C., Rosa, M. J., Rao, A., Bebko, G., Bertocci, M. A., et al. (2016). Can emotional and behavioral dysregulation in youth be decoded from functional neuroimaging? PLOS ONE, 11(1):e0117603.

Regier, D. A., Farmer, M. E., Rae, D. S., Locke, B. Z., Keith, S. J., et al. (1990). Comorbidity of mental disorders with alcohol and other drug abuse: results from the Epidemiologic Catchment Area (ECA) study. JAMA, 264(19):2511-2518.

Saxe, G. N., Ma, S., Ren, J., and Aliferis, C. (2017). Machine learning methods to predict child posttraumatic stress: a proof of concept study. BMC Psychiatry, 17(1):223.

Shalev, A. Y., Sahar, T., Freedman, S., Peri, T., Glick, N., et al. (1998). A prospective study of heart rate response following trauma and the subsequent development of posttraumatic stress disorder. Archives of General Psychiatry, 55(6):553-559.

Weathers, F. W., Litz, B. T., Herman, D. S., Huska, J. A., Keane, T. M., et al. (1993). The PTSD Checklist (PCL): Reliability, validity, and diagnostic utility. In Annual Convention of The International Society for Traumatic Stress Studies, San Antonio, TX, volume 462. San Antonio, TX.

Weathers, F. W., Litz, B. T., Keane, T. M., Palmieri, P. A., Marx, B. P., et al. (2013). The PTSD checklist for DSM-5 (PCL-5). Scale available from the National Center for PTSD at www.ptsd.va.gov.

Witten, I. H., Frank, E., Hall, M. A., and Pal, C. J. (2016). Data Mining: Practical machine learning tools and techniques. Morgan Kaufmann, 4 edition. 